\title{
New perspective on hybrid mesons
}

\author{
Shu-Sheng $\mathrm{Xu}^{1}$, Zhu-Fang Cui ${ }^{2}$, Lei Chang ${ }^{3, \text { a }}$, Joannis Papavassiliou ${ }^{4}$, Craig D. Roberts ${ }^{5, b}$, and Hong-Shi Zong ${ }^{2, c}$ \\ 1 School of Science, Nanjing University of Posts and Telecommunications, Nanjing, Jiangsu 210023, China \\ 2 School of Physics, Nanjing University, Nanjing, Jiangsu 210093, China \\ 3 School of Physics, Nankai University, Tianjin 300071, China \\ 4 Department of Theoretical Physics and IFIC, University of Valencia and CSIC, E-46100, Valencia, Spain \\ 5 Physics Division, Argonne National Laboratory, Argonne, IL 60439, USA
}

Received: 12 June 2019

Published online: 22 July 2019

(C) The Author(s) 2019. This article is published with open access at Springerlink.com

Communicated by A. Peshier

\begin{abstract}
We introduce a novel approach to the hybrid-meson (valence-gluon+quark+antiquark) boundstate problem in relativistic quantum field theory. Exploiting the existence of strong two-body correlations in the gluon-quark, $q_{g}=[g q]$, and gluon-antiquark, $\bar{q}_{g}=[g \bar{q}]$ channels, we argue that a sound description of hybrids can be obtained by solving a coupled pair of effectively two-body equations; and, consequently, that hybrids may be viewed as highly correlated $q_{g} \bar{q} \leftrightarrow q \bar{q}_{g}$ bound states. Analogies may be drawn between this picture of hybrid structure and that of baryons, in which diquark (quark+quark) correlations play a key role. The potential of this formulation is illustrated by calculating the spectrum of light-quark isovector hybrid mesons.
\end{abstract}

\section{Introduction}

The known spectrum of light hadrons is simple in the sense that it qualitatively matches the pattern established by constituent-quark models [1,2]: there are mesons built from a constituent-quark-antiquark $(Q \bar{Q})$ pair and baryons constituted from three constituent quarks $(Q Q Q)$, where $Q$ is associated with any one of the light $u-, d-, s$-quarks. Notably, refs. [1,2] also raised the possibility that more complicated hadrons are possible, e.g. $Q Q \bar{Q} \bar{Q}$ and $Q \bar{Q} Q Q Q$. No candidates were then known; but after fifty years, in systems involving the heavier $c$ - and $b$-quarks, that has now changed $[3-5]$.

Following the emergence of quantum chromodynamics (QCD) more than forty years ago, from an array of distinct ideas and discoveries [6,7], new possibilities arose. As a non-Abelian quantum gauge field theory, in which eight self-interacting gauge bosons (gluons) mediate the interactions between current quarks, QCD can conceivably support systems with valence glue, e.g. hybrid mesons, $Q \bar{Q} G$; hybrid baryons, $Q Q Q G$; and even glueballs, $G G$. Here, $G$ is a nebulously defined "constituent gluon" degree of freedom, whose nature will only become known once such systems are detected experimentally. Meanwhile, today's tabulations of hadron masses identify at least three plausible hybrid-meson

\footnotetext{
a e-mail: leichang@nankai.edu.cn

b e-mail: cdroberts@anl.gov

c e-mail: zonghs@nju.edu.cn
}

candidates below $2 \mathrm{GeV}$ [8], and dedicated searches for such states are a worldwide priority, e.g. refs. $[9,10]$.

Many models have been used to calculate the spectrum of light hybrid mesons $[3-5,11,12]$ : the approaches are distinguished by, inter alia, their disparate treatments/definitions of $G$; and the resulting spectra disagree. Nevertheless, development of a reliable continuum method for calculating hybrid meson properties would be valuable, primarily for the interpretation of empirical observations but also to provide insights into results obtained via lattice-regularised QCD (lQCD) $[13,14]$.

$Q \bar{Q}$ mesons in quantum mechanics cannot possess the following (exotic) quantum numbers [8]: $J^{P C}=0^{+-}$, $0^{--}, 1^{-+}$, etc. This is not true in Poincaré-covariant treatments of two-valence-body bound states owing to the existence of an additional degree of freedom, i.e. the relative time between the valence-quark and -antiquark [15-17]. However, extant studies of exotic mesons using truncations of the Bethe-Salpeter kernel produce unrealistic spectra [16-22], viz. exotic mesons with masses so light that they should already have been seen when, in fact, signals for such states are currently weak and lie at significantly higher masses. Furthermore, the two-body Bethe-Salpeter equation does not readily entertain a distinction between regular mesons and hybrids with the same $J^{P C}$. These weaknesses are not remedied when more sophisticated two-body kernels [23-27] are used; and we judge them to be a signal that hybrid mesons must contain an explicit valence-gluon degree of freedom. 


\section{Faddeev equation for hybrid mesons}

Given the preceding observations, herein we formulate a Poincaré-covariant Faddeev equation for hybrids, which treats these systems as bound states of a valence-gluon, -quark and -antiquark. Importantly, each of these valence constituents is strongly dressed, e.g. possessing an emergent mass that is large at infrared momenta [28].

Our starting point is an observation [27] that the standard inhomogeneous Bethe-Salpeter equation for the gluon-quark vertex can be rewritten in terms of a gluonquark (Compton) scattering amplitude, $C$, which is one particle irreducible in the $s$-channel:

$$
\begin{aligned}
& \Gamma_{\mu}^{a}(k, q)=\gamma_{\mu} t^{a}+\tilde{\Gamma}_{\mu}^{a}(k, q), \\
& \tilde{\Gamma}_{\mu}^{a}(k, q)=\int_{d \ell}^{\Lambda} \gamma_{\rho} t^{b} S\left(\ell_{+}\right) D_{\rho \sigma}\left(\bar{\ell}_{-}\right) C_{\sigma \mu}^{b a}(\ell, q ; k),
\end{aligned}
$$

where $\left\{t^{a}=\lambda^{a} / 2, a=1, \ldots, 8\right\}$, with $\left\{\lambda^{a}\right\}$ the $S U(3)$ colour Gell-Mann matrices; $\int_{d \ell}^{\Lambda}$ represents a Poincaré invariant regularisation of the four-dimensional integral, with $\Lambda$ the regularization mass-scale; and $S, D_{\rho \sigma}$ are the dressed-quark and -gluon propagators, respectively, with $\ell_{ \pm}=\ell+\eta k, \bar{\ell}_{ \pm}=\ell \pm(1-\eta) k, \eta \in[0,1]$. Applying charge conjugation, one obtains an equivalent equation for gluonantiquark scattering.

Recalling textbook derivations of the two-body BetheSalpeter equation in analyses of two-particle scattering and the relationship between the scattering matrix and kernel; and also the role that coloured quark-quark (diquark) correlations play in simplifying the baryon threebody problem [29-37], then eq. (1b) suggests the possibility that analogous gluon-quark $[g q]$ and degenerate gluonantiquark $[g \bar{q}]$ correlations may play a material role in solving the three-body problem for hybrid mesons.

Supposing that strong $g q$ and $g \bar{q}$ correlations exist, then the bound-state equation depicted in fig. 1 should provide the basis for a sound description of hybrid mesons ${ }^{1}$. Here the complete bound-state amplitude is $\Psi=\Psi_{1}+\Psi_{2}$, with $\Psi_{1,2}$ describing the $\left[q_{g} \bar{q}\right],\left[q \bar{q}_{g}\right]$ relative momentum correlations, respectively:

$$
\Psi_{1 \mu}^{a}(p, l ; P)=\Gamma_{\mu}^{a}\left(l ; p_{q_{g}}\right) \mathcal{S}_{g}\left(p_{q_{g}}\right) \psi_{1}(p ; P),
$$

where $\Gamma_{\mu}^{a}\left(\ell ; p_{q_{g}}\right)$ is the canonically normalised amplitude [38] describing a $[g q]$ correlation with total momentum $p_{q_{g}}$ and relative momentum $l$, which has the matrix structure of a gluon-quark vertex; $\mathcal{S}$ is the propagator for this correlation; and $\psi_{1}(p ; P)$ is a meson-like BetheSalpeter amplitude. $\Psi_{2}$ is plain by analogy. (We retain only the lightest $g q$ and $g \bar{q}$ correlations in this first analysis.)

Focusing on states with good charge-conjugation parity, such as isovector hybrids in the isospin-symmetry

\footnotetext{
1 Notably, this approach forces the valence-quark and valence-antiquark within the hybrid meson to exist in a relative colour-octet configuration: a singlet $q \bar{q}$ configuration cannot combine with a valence-gluon (colour-octet) to produce an overall colour-singlet system.
}

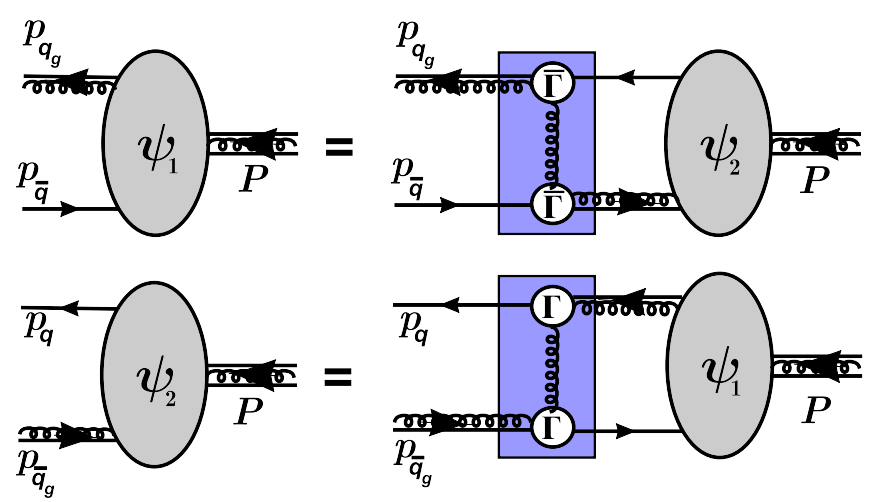

Fig. 1. Poincaré covariant Faddeev equation for a $g q \bar{q}$ hybrid with total momentum $P=p_{q_{g}}-p_{\bar{q}}=p_{q}+p_{g}-p_{\bar{q}}=p_{q}-p_{\bar{q}_{g}}$, obtained assuming that strong $g q$ and $g \bar{q}$ correlations exist within this three-valence-body system. The complete bound-state amplitude is $\Psi(P)=\Psi_{1}(P)+\Psi_{2}(P)$; and the coupled-channels problem simplifies to eq. (3) for states with good charge-conjugation parity. The rectangles demarcate the active kernels of this Faddeev equation — single line: dressed quark/antiquark propagator; adjacent line and spring (coiled line): $\mathcal{S}_{g}$, propagators for the $q_{g}=[g q], \bar{q}_{g}=[g \bar{q}]$ correlations; $\Gamma, \bar{\Gamma}: q_{g}$, $\bar{q}_{g}$ correlation amplitudes, respectively; and single spring: $D$, propagator of the gluon that leaves $\bar{q}_{g}$ to combine with $q$, forming $q_{g}$, and vice versa.

limit, then $\psi_{1,2}(p ; P)=\bar{\psi}_{2,1}(p ; P)=p \psi_{2,1}(p ; P), p=$ \pm 1 , where $\bar{\psi}(p ; P)=C[\psi(-p ; P)]^{\mathrm{T}} C^{\dagger}$ with $C=\gamma_{2} \gamma_{4}$ the charge-conjugation matrix and $[\cdot]^{\mathrm{T}}$ indicating matrix transpose. Hence, for such systems, the solution of the coupled-channel Faddeev equation in fig. 1 is completely determined by the following Bethe-Salpeter equation:

$$
\begin{aligned}
\psi_{2}(p ; P)= & p \int_{d \ell} D_{\mu \nu}\left(p_{+}-\bar{\ell}_{+}\right) \Gamma_{\mu}^{a}\left(p_{+}-\frac{1}{2} \bar{\ell}_{+} ; \bar{\ell}_{+}\right) \mathcal{S}_{g}\left(\bar{\ell}_{+}\right) \\
& \times \bar{\psi}_{2}(\ell ; P) S\left(\ell_{-}\right) \Gamma_{\nu}^{a}\left(\ell_{-}-\frac{1}{2} \bar{p}_{-} ; \bar{p}_{-}\right)
\end{aligned}
$$

The challenges now are to confirm the existence of tight gluon-quark correlations and determine their properties.

\section{Gluon-quark correlation}

Adapting the logic used to establish the existence and properties of diquark correlations [32-34], we search for a pole solution to a leading-order (rainbow-ladder [27]) truncation of eq. (1), i.e. for a solution of the following homogeneous Bethe-Salpeter equation, $\Gamma_{\mu}^{a}=t^{a} \Gamma_{\mu}, k=p-\ell$ :

$$
\begin{aligned}
& t^{a} \Gamma_{\mu}(p ; Q) \Lambda_{+}=-\int_{d \ell} \tilde{\mathcal{G}}\left(k^{2}\right) t^{b} \gamma_{\rho} S\left(\ell_{+}\right) \\
& \times t^{c} \Gamma_{\lambda}(\ell ; Q) D_{\lambda \tau}\left(\bar{\ell}_{-}\right) f_{3 g}\left(k^{2}\right){ }_{0} V_{\rho \tau \mu}^{b c a}\left(k, \bar{\ell}_{-}, \bar{p}_{-}\right) \Lambda_{+},
\end{aligned}
$$

where $\Lambda_{+}=\left[-i \gamma \cdot Q+m_{g q}\right] /\left[2 m_{g q}\right]$, with $m_{g q}$ the boundstate mass, is the positive-energy projection operator. 
Here [19,20]

$$
\frac{1}{Z_{2}^{2}} \tilde{G}\left(k^{2}\right)=\frac{8 \pi^{2}}{\omega^{4}} D \mathrm{e}^{-k^{2} / \omega^{2}}+\frac{8 \pi^{2} \gamma_{m} \mathcal{E}\left(k^{2}, m_{T}^{2}\right)}{\ln \left[\tau+\left(1+k^{2} / \Lambda_{\mathrm{QCD}}^{2}\right)^{2}\right]},
$$

with $\gamma_{m}=12 / 25, \Lambda_{\mathrm{QCD}}=0.234 \mathrm{GeV}, \tau=\mathrm{e}^{2}-1$, $\mathcal{E}\left(k^{2}, m_{T}^{2}\right)=\left[1-\exp \left(-k^{2} / m_{T}^{2}\right)\right] / k^{2}, m_{T}=2 \omega$, is chosen because it is the basis for a successful description of a wide variety of hadron phenomena; ${ }_{0} V_{\rho \tau \mu}^{b c a}$ is the bare three-gluon vertex; and $f_{3 g}\left(k^{2}\right)=\left[1-\exp \left(-k^{2} / \omega_{3 g}^{2}\right)\right]$, with $\omega_{3 g}$ a parameter, is included so that the active three-gluon vertex exhibits the infrared suppression found in modern studies [39-42]. $Z_{2}$ is the dressedquark wave function renormalisation constant: we use a momentum-subtraction scheme, with renormalisation at $\zeta=19 \mathrm{GeV}$ [43]. (A quark exchange diagram can also contribute to the kernel of eq. (4) at this order; but it is numerically sub-leading and therefore neglected.)

In order to solve eq. (4), we first observe that the solution has the form

$$
\begin{aligned}
\Gamma_{\mu}(p ; Q) & =\sum_{i=1}^{6} g_{i}(p ; Q) t_{\mu}^{i}(p, Q), \\
t^{1} & =\gamma_{\mu}, \quad t^{2}=i \hat{p}_{\mu}, \quad t^{3}=\hat{Q}_{\mu}, \\
t^{4} & =i \gamma \cdot \hat{p} \hat{Q}_{\mu}, \quad t^{5}=i \gamma_{\mu} \gamma \cdot \hat{p}, \quad t^{6}=\gamma \cdot \hat{p} \hat{p}_{\mu},
\end{aligned}
$$

with $\hat{p}^{2}=1, \hat{Q}^{2}=-1$, and $\left\{g_{i}, i=1, \ldots, 6\right\}$ are scalar functions. Inserting eq. (6a) into eq. (4) yields a coupledchannels eigenvalue problem for the correlation's amplitude functions, $\left\{g_{i}\right\}$, and mass-squared, $Q^{2}=-m_{g q}^{2}$. Following ref. [43], such equations are now readily solved, and we adapt algorithms from ref. [44] when necessary.

Completing the kernel of eq. (4) with $\omega=0.5 \mathrm{GeV}$, $D \omega=(0.96 \mathrm{GeV})^{3}$, values determined by fitting an array of $\pi$ - and $\rho$-meson observables, with renormalisation group invariant current-quark mass $\hat{m}=6.3 \mathrm{MeV} ; S$ used in calculating those meson results, obtained from the consistent dressed-quark gap equation; $\omega_{3 g}=0.9 \mathrm{GeV}$; and a dressed propagator for the gluon constituent in the form $D_{\lambda \tau}\left(\bar{\ell}_{-}\right)=\delta_{\lambda \tau} \mathcal{E}\left(\bar{\ell}_{-}^{2}, m_{g}^{2}\right), m_{g}=0.6 \mathrm{GeV}$, one finds a solution of eq. (6a) with $m_{g q}=1.0 \mathrm{GeV}\left(=m_{g \bar{q}}\right)$. The existence of a solution is robust, e.g. almost identical results are obtained using the separable model in refs. [16,17].

A result $m_{g q} \simeq 1 \mathrm{GeV}$ is natural because dressed lightquark propagation is characterised by an infrared massscale $M \simeq 0.35 \mathrm{GeV}$ [45-47]; a dressed-gluon, which couples strongly to a quark-antiquark pair, should have a mass $\simeq 2 M$, which is roughly the value we have chosen and consistent with that computed elsewhere [48]; and the composite $g q$ system should thus have a mass $\simeq 3 M$. Notably, under combined $\pm 10 \%$ variations in $\omega_{3 g}$ and $m_{g}$, $m_{g q} \rightarrow(1 \pm 0.1) m_{g q}$.

This analysis reveals that, as in colour-antitriplet diquark channels, interactions that deliver a good description of ground-state hadron properties also generate strong $[g q]$ correlations. They should be confined, but this is not true in RL truncation. Notably, corrections to this simplest approximation are critical in diquark channels, serving to eliminate bound-state poles from the quarkquark scattering matrix whilst simultaneously preserving the strong diquark correlations [49]. Such corrections should similarly affect $[g q]$ correlations.

\section{Hybrid meson spectrum}

Now that eq. (3) is validated, we solve it for the spectrum of light-quark ground-state hybrids. Regarding the kernel, having already solved eq. (4), the dressed-quark and -gluon propagators, $S, D_{\mu \nu}$, respectively, and canonicallynormalised $[g q]$ correlation amplitudes, $\Gamma_{\nu}^{a}$, are all in hand. The remaining element is $\mathcal{S}_{g}$. Equations (1) reveal that a quark-gluon correlation must, in all practical aspects, behave like a dressed quark: it is a colour-triplet fermion-like object whose propagator takes the standard form:

$$
S_{g}(\ell)=-i \gamma \cdot \ell \sigma_{V}\left(\ell^{2}\right)+\sigma_{S}\left(\ell^{2}\right) .
$$

One could develop a gap equation for this propagator, which would determine the scalar functions $\sigma_{V, S}$; but that would merely amount to a complicated model. Herein, we choose instead to be led by experience with dressed quarks and diquark correlations in the baryon problem, and simplify the analysis by employing the following Ansätze $\left(s=\ell^{2}\right)[50,51]$ :

$$
\sigma_{V}(s)=\mathcal{E}\left(s, s_{V}\right), \quad \sigma_{S}(s)=\frac{m_{g q}}{s}\left[1-s_{S} \mathcal{E}\left(s, s_{S}\right)\right],
$$

where the parameters $s_{V, S}$ are described in connection with eq. (9) below. These forms ensure that $S_{g}$ may be understood to describe a confined excitation owing to the associated violation of reflection positivity [52].

As is typical of Faddeev equations involving composite degrees of freedom, the $[g q]$ correlation amplitudes in eq. (3) are sampled off-shell and the integrand therefore exhibits spurious ultraviolet behaviour unless one introduces an off-shell extension of the basis in eq. (6b). Adapting the procedure in ref. [53], we write $\hat{Q}=Q h\left(Q^{2}\right) /\left[i m_{g q}\right], t^{i \neq 1} \rightarrow t^{i \neq 1} h\left(Q^{2}\right)^{2}$, where $h\left(Q^{2}\right)^{2}=$ $m_{g q}^{2} /\left[Q^{2}+2 m_{g q}^{2}\right]$. Evidently, $h\left(Q^{2}=-m_{g q}^{2}\right)=1$.

The kernel of eq. (3) is now complete; and using the approach just described and the full bound-state amplitude in all cases $[15,18]$, we solved eq. (3) for the ground-state masses and on-shell amplitudes in the following five lightquark isovector channels: $J^{P C}=0^{-+}, 1^{--}, 0^{+-}, 0^{--}$, $1^{-+}$, the last three of which are quark model exotics. (We used $\eta=1 / 3$ because it simplifies the numerical analysis and the masses do not depend on this choice when Poincaré-covariance is preserved throughout.) The spectrum thus obtained is listed in Row 1 of table 1 . Bound states exist in all channels with, notably, the $0^{-+}, 1^{--}$ hybrids being structurally distinct from those accessible using the two-body Bethe-Salpeter equation in the channels. However, in comparison with lQCD predictions [13], almost all states are too light, especially $0^{-+}$, and the $1^{-+} 1^{--}$ordering is reversed. Wide variations of $m_{g q}$, $s_{V, S}$, do not alter this outcome. 
Table 1. Row 1: hybrid-meson masses obtained from eq. (3). Under combined $\pm 10 \%$ changes of $m_{q g}$ and $s_{V, S}$ in eq. (8), these results have the indicated sensitivity. Row 2 : hybrid spectrum obtained after enhancing the anomalous chromomagnetic moment term in the $[g q]$ correlation amplitude, see eq. (9): $\kappa_{g q} \rightarrow \kappa_{g q}(1 \pm 0.1)$ has negligible impact. The 1 QCD results in Rows 5 and 6 were computed on dynamical lattices with pion masses $m_{\pi} \approx(0.52,0.44,0.40) \mathrm{GeV}$ and two volumes $16^{3} \times 128$, $20^{3} \times 128$ [13]. These configurations yield a mass for the pion's first radial excitation that is approximately $\delta_{\pi_{1}}=0.43 \mathrm{GeV}$ larger than experiment [8]. In Rows 3 and 4, we renormalise the lQCD results by subtracting $\delta_{\pi_{1}}$. (All tabulated results listed in $\mathrm{GeV}$.)

\begin{tabular}{l|ccccc}
\hline & $0^{-+}$ & $1^{-+}$ & $1^{--}$ & $0^{+-}$ & $0^{--}$ \\
\hline RL direct & $1.28(9)$ & $1.80(4)$ & $1.64(10)$ & $1.73(13)$ & $1.74(3)$ \\
ACM improved & $1.62(6)$ & $1.75(8)$ & $1.86(10)$ & $1.87(14)$ & $1.90(3)$ \\
\hline lQCD $_{R}-16^{3}$ & $1.72(2)$ & $1.73(2)$ & $1.84(2)$ & $2.03(1)$ & \\
lQCD $_{R}-20^{3}$ & $1.69(2)$ & $1.72(2)$ & $1.77(6)$ & $1.99(2)$ & \\
lQCD $-16^{3}$ & $2.14(1)$ & $2.15(2)$ & $2.26(2)$ & $2.45(1)$ & \\
lQCD $-20^{3}$ & $2.12(2)$ & $2.16(2)$ & $2.21(6)$ & $2.43(2)$ & \\
\hline
\end{tabular}

The mismatch between the spectrum in Row 1 of table 1 and lQCD results caused us to reconsider each element in our formulation of the hybrid meson problem. Drawing upon analyses of improvements to RL truncation [23-25], we were led to the probable origin. Namely, the $[g q]$ correlation amplitude was computed in RL truncation and, consequently, the anomalous chromomagnetic moment (ACM) associated with this correlation is greatly underestimated owing to the failure of RL truncation to reliably express effects of dynamical chiral symmetry breaking [24] (DCSB or, equivalently, emergence of strong mass). We therefore omitted the spin-independent coupling $t^{3}$, multiplied the ACM related term in eq. (6b), $t^{5}$, by a parameter, $\kappa_{g q}$, and varied $\left(\kappa_{g q}, s_{V, S}\right)$ in search of a triplet that reproduces the sign of the $1^{-+}-1^{--}-0^{+-}$ lQCD splittings. Notably, no constraint was placed on the value of any single mass. The fact that a solution to this problem does exist:

$$
\kappa_{g q}=2.4, \quad s_{V}=(0.70 \mathrm{GeV})^{2}, \quad s_{S}=(0.63 \mathrm{GeV})^{2},
$$

supports our conjecture regarding the origin of the mismatch; and these values generate the spectrum in Row 2 of table 1. (This analysis suggests that no solution of the valence-gluon+quark+antiquark Faddeev equation which adheres strictly to the leading-order RL truncation can produce a realistic spectrum of hybrids.)

Figure 2 compares the lattice results in Rows 3, 4 of table 1 with our ACM-improved calculations in Row 2. The level ordering is identical. Furthermore, the absolute values of the masses are commensurate. This is a nontrivial outcome. The lQCD masses plotted in fig. 2 are rescaled by subtraction of $\delta_{\pi_{1}}$, the difference between the simulation results for the mass of the pion's first radial excitation and its empirical value, a number which is completely unrelated to our calculations. Moreover, as remarked above, no single lQCD mass was used as a constraint when fitting



Fig. 2. Comparison between our ACM-improved spectrum (stars, green), Row 2 in table 1, and the rescaled lQCD results in Rows 3 (up-triangles, red) and 4 (down-triangles, blue).

the parameters in eq. (9). The magnitude of our results is instead set by the infrared values of the running gluon and quark masses, which are determined by $\pi$ - and $\rho$-meson properties, unrelated to hybrid channels.

The $0^{--}$state deserves additional attention. IQCD predicts this channel to host the heaviest light-quark hybrid ground state, with the lightest such meson lying more than $2 \mathrm{GeV}$ above the ground state $\rho$-meson [13]. The Faddeev equation in fig. 1, which capitalises on the existence and properties of $[g q],[g \bar{q}]$ correlations in $g q \bar{q}$ scattering, confirms that the $0^{--}$is the heaviest hybrid, thereby correcting a defect of RL-truncation analyses of exotics using the two-body Bethe-Salpeter equation [1922 . Our computed $0^{--}$mass might nevertheless be too light because such a system probably possesses a large amount of angular momentum [54], leading to significant DCSB-enhanced repulsion within the bound-state; and our simple expedient for ameliorating the associated defects of RL truncation may not be completely adequate. The approach we have described will always produce a heavy $0^{--}$state, but its precise location must await future, more sophisticated analyses.

\section{Epilogue}

We introduced a novel approach to the valencegluon+quark+antiquark bound-state problem in relativistic quantum field theory. Beginning with the associated Faddeev equation, we demonstrated that strong correlations exist in the gluon-quark, $q_{g}=[g q]$, and gluonantiquark, $\bar{q}_{g}=[g \bar{q}]$ channels; and hence that a simpler, coupled pair of effectively two-body equations can provide the basis for a realistic description of hybrid mesons (fig. 1). Consequently, hybrid mesons appear as highlycorrelated $q_{g} \bar{q} \leftrightarrow q \bar{q}_{g}$ bound-states. In hindsight, given the role that coloured quark+quark correlations play in determining the properties of the neutron, proton, and other baryons, the existence and importance of kindred correlations within hybrids appears credible. 
To illustrate the potential of this formulation, we employed a simple model to describe the properties of the $[g q],[g \bar{q}]$ correlations and therewith established that it can readily reproduce the location and ordering of groundstate light-quark hybrids obtained via the numerical simulation of lattice-regularised QCD. This outcome distinguishes our approach from other continuum frameworks. Notably, an understanding of the impact of emergent strong-interaction mass in forming the $[g q],[g \bar{q}]$ correlations is crucial to this success.

Ours is a first analysis of hybrid mesons from this new perspective. More sophisticated treatments are necessary before the validity of the formulation can firmly be established. Meanwhile, it should serve as a guide for subsequent continuum treatments of the hybrid-meson threebody problem; and the highly correlated wave functions it yields can be used to predict a range of hybrid decays and other processes in order to elucidate empirical signatures for the presence and role of $[g q],[g \bar{q}]$ correlations.

We are grateful for constructive suggestions from D. Binosi, S.X. Qin and J. Segovia. Work supported by China Postdoctoral Science Foundation (under Grant No. 2016M591809); Chinese Government Thousand Talents Plan for Young Professionals; Generalitat Valenciana under grant Prometeo II/2014/066; Jiangsu Province Hundred Talents Plan for Professionals; Jiangsu Province Natural Science Foundation of China (under Grant No. BK20180323); National Natural Science Foundation of China (under Grant Nos. 11535005, 11690030, 11805097 and 11847024); Nanjing University of Posts and Telecommunications Science Foundation (Grant No. NY219032); Spanish MEyC, under grants FPA2017-84543-P and SEV-2014-0398; U.S. Department of Energy, Office of Science, Office of Nuclear Physics, under contract No. DE-AC02-06CH11357.

Data Availability Statement This manuscript has no associated data or the data will not be deposited. [Authors' comment: All data generated during this study are contained in this published article.]

Publisher's Note The EPJ Publishers remain neutral with regard to jurisdictional claims in published maps and institutional affiliations.

Open Access This is an open access article distributed under the terms of the Creative Commons Attribution License (http://creativecommons.org/licenses/by/4.0), which permits unrestricted use, distribution, and reproduction in any medium, provided the original work is properly cited.

\section{References}

1. M. Gell-Mann, Phys. Lett. 8, 214 (1964).

2. G. Zweig, An SU(3) model for strong interaction symmetry and its breaking. Parts 1 and 2, CERN Reports No. 8182/TH. 401 and No. 8419/TH. 412 (1964).

3. R.F. Lebed, R.E. Mitchell, E.S. Swanson, Prog. Part. Nucl. Phys. 93, 143 (2017).
4. A. Ali, J.S. Lange, S. Stone, Prog. Part. Nucl. Phys. 97, 123 (2017).

5. S.L. Olsen, T. Skwarnicki, D. Zieminska, Rev. Mod. Phys. 90, 015003 (2018).

6. W.J. Marciano, H. Pagels, Phys. Rep. 36, 137 (1978).

7. W.J. Marciano, H. Pagels, Nature 279, 479 (1979).

8. C. Patrignani et al., Chin. Phys. C 40, 100001 (2016).

9. F. Nerling, EPJ Web of Conferences 126, 04033 (2016) Highlights from the COMPASS experiment at CERN.

10. S. Dobbs, Searching for Hybrid Mesons with GlueX, PoS (Hadron 2017) 047 (2018).

11. C.A. Meyer, E.S. Swanson, Prog. Part. Nucl. Phys. 82, 21 (2015).

12. J.M. Richard, Few Body Syst. 57, 1185 (2016).

13. J.J. Dudek, R.G. Edwards, M.J. Peardon, D.G. Richards, C.E. Thomas, Phys. Rev. D 82, 034508 (2010).

14. J.J. Dudek, Phys. Rev. D 84, 074023 (2011).

15. C.H. Llewellyn-Smith, Ann. Phys. 53, 521 (1969).

16. C.J. Burden, L. Qian, C.D. Roberts, P.C. Tandy, M.J. Thomson, Phys. Rev. C 55, 2649 (1997).

17. C.J. Burden, M.A. Pichowsky, Few Body Syst. 32, 119 (2002).

18. A. Krassnigg, Phys. Rev. D 80, 114010 (2009).

19. S.X. Qin, L. Chang, Y.X. Liu, C.D. Roberts, D.J. Wilson, Phys. Rev. C 84, 042202(R) (2011).

20. S.X. Qin, L. Chang, Y.X. Liu, C.D. Roberts, D.J. Wilson, Phys. Rev. C 85, 035202 (2012).

21. C.S. Fischer, S. Kubrak, R. Williams, Eur. Phys. J. A 50, 126 (2014).

22. T. Hilger, A. Krassnigg, Eur. Phys. J. A 53, 142 (2017).

23. L. Chang, C.D. Roberts, Phys. Rev. Lett. 103, 081601 (2009).

24. L. Chang, Y.X. Liu, C.D. Roberts, Phys. Rev. Lett. 106, 072001 (2011).

25. L. Chang, C.D. Roberts, Phys. Rev. C 85, 052201(R) (2012).

26. R. Williams, C.S. Fischer, W. Heupel, Phys. Rev. D 93, 034026 (2016).

27. D. Binosi, L. Chang, S.X. Qin, J. Papavassiliou, C.D. Roberts, Phys. Rev. D 93, 096010 (2016).

28. C.D. Roberts, Few Body Syst. 59, 72 (2018).

29. R.T. Cahill, C.D. Roberts, J. Praschifka, Aust. J. Phys. 42, 129 (1989).

30. C.J. Burden, R.T. Cahill, J. Praschifka, Aust. J. Phys. 42, 147 (1989).

31. R.T. Cahill, Aust. J. Phys. 42, 171 (1989).

32. R.T. Cahill, C.D. Roberts, J. Praschifka, Phys. Rev. D 36, 2804 (1987).

33. P. Maris, Few Body Syst. 32, 41 (2002).

34. P. Maris, Few Body Syst. 35, 117 (2004).

35. H. Reinhardt, Phys. Lett. B 244, 316 (1990).

36. G.V. Efimov, M.A. Ivanov, V.E. Lyubovitskij, Z. Phys. C 47, 583 (1990).

37. G. Eichmann, R. Alkofer, A. Krassnigg, D. Nicmorus, Phys. Rev. Lett. 104, 201601 (2010).

38. N. Nakanishi, Prog. Theor. Phys. Suppl. 43, 1 (1969).

39. A.C. Aguilar, D. Binosi, D. Ibañez, J. Papavassiliou, Phys. Rev. D 89, 085008 (2014).

40. A. Athenodorou, D. Binosi, P. Boucaud, F. De Soto, J. Papavassiliou, J. Rodríguez-Quintero, S. Zafeiropoulos, Phys. Lett. B 761, 444 (2016).

41. P. Boucaud, F. De Soto, J. Rodríguez-Quintero, S. Zafeiropoulos, Phys. Rev. D 95, 114503 (2017). 
42. L. Corell, A.K. Cyrol, M. Mitter, J.M. Pawlowski, N. Strodthoff, Correlation functions of three-dimensional Yang-Mills theory from the FRG, arXiv:1803.10092 [hep$\mathrm{ph}$.

43. P. Maris, C.D. Roberts, Phys. Rev. C 56, 3369 (1997).

44. A. Krassnigg, PoS (Confinement) 8, 075 (2008).

45. M.S. Bhagwat, M.A. Pichowsky, C.D. Roberts, P.C. Tandy, Phys. Rev. C 68, 015203 (2003).

46. P.O. Bowman et al., Phys. Rev. D 71, 054507 (2005).

47. M.S. Bhagwat, P.C. Tandy, AIP Conf. Proc. 842, 225 (2006).

48. D. Binosi, C.D. Roberts, J. Rodríguez-Quintero, Phys. Rev. D 95, 114009 (2017).
49. M.S. Bhagwat, A. Höll, A. Krassnigg, C.D. Roberts, P.C. Tandy, Phys. Rev. C 70, 035205 (2004).

50. R. Alkofer, A. Höll, M. Kloker, A. Krassnigg, C.D. Roberts, Few Body Syst. 37, 1 (2005).

51. J. Segovia, I.C. Cloët, C.D. Roberts, S.M. Schmidt, Few Body Syst. 55, 1185 (2014).

52. T. Horn, C.D. Roberts, J. Phys. G 43, 073001 (2016).

53. G. Eichmann, A. Krassnigg, M. Schwinzerl, R. Alkofer, Ann. Phys. 323, 2505 (2008).

54. M. Berwein, N. Brambilla, J. Tarrús Castellà, A. Vairo, Phys. Rev. D 92, 114019 (2015). 